\title{
Videourodynamic assessment of diurnal urinary incontinence
}

\author{
M BORZYSKOWSKI AND A R MUNDY
}

Departments of Paediatrics, Urology, and Surgery, Guy's Hospital, London

SUMMARY Persistent daytime incontinence is a fairly uncommon but important problem. Videourodynamic studies were carried out in 215 children presenting with this problem in whom there were no neurological signs and who had not responded to simple measures. Over $90 \%$ of symptomatic children were shown to have an abnormality, of which over half had detrusor instability and a fifth had the wide bladder neck anomaly. We consider that it is important that these children are fully assessed as many can be helped.

Children with diurnal wetting may be referred to a general paediatrician, paediatric neurologist, paediatric nephrologist, urologist, or psychiatrist. Most of these children have never been reliably dry by day, although occasionally the symptom is of recent onset. They are usually treated empirically.

The advent of the videourodynamic study has allowed us to assess bladder and urethral function more accurately. This investigation has proved invaluable in the assessment and management of the child with a neuropathic bladder and has enabled us to identify those children at high risk of renal damage. ${ }^{1}$

The study consists of a filling and voiding cystometrogram combined with a micturating cystourethrogram. ${ }^{2}$ They are recorded simultaneously onto video tape and are viewed side by side using a split screen display so that events occurring in the bladder and urethra can be correlated with pressure changes. As far as the child is concerned measurement of rectal pressure is the only additional feature when compared with a micturating cystourethrogram. A great deal more information, however, is obtained.

\section{Patients and methods}

We have reviewed the urodynamic findings in 215 children (with an age range of 3.5 to 16 years) studied at this hospital over the last six years. The children were selected for study because of persistent diurnal wetting that had not responded to simple measures such as star charts and timed voiding. Most wet their pants at school on most days and some had to take changes of clothing to school daily. Children with an ectopic ureter were excluded.

All were physically normal and none had neurological signs. Children with nocturnal enuresis alone were not included; indeed, we rarely study these children as it has been found that most have normal bladder function. ${ }^{3}$ A careful history is important, however, as it may reveal daytime problems that have not been volunteered by the parent or child.

The studies were all performed using standard urodynamic techniques and urethral catheters. The children were not sedated and failure to carry out a study because of lack of cooperation occurred in less than $5 \%$.

Measurement of urinary flow rate was included as a standard part of the study and provided useful additional information where there was evidence of obstruction to urinary flow-for example, distal urethral stenosis-particularly if high detrusor pressures were being generated in an effort to try and overcome this. If there was a low urinary volume, however, then the flow rate would be reduced. The flow rate could also be measured independently and could thus be checked if it was thought that inhibition had affected this during the study itself.

\section{Results}

As can be seen in Table 1 over $90 \%$ of the children were found to have an abnormality and in over half this was due to detrusor instability. A fifth of the children had the wide bladder neck anomaly. Some had more than one abnormality, the commonest 
Table 1 Videourodynamic abnormalities found in 215 children with diurnal enuresis

\begin{tabular}{lc}
\hline Detrusor instability & $123(57 \%)$ \\
Wide bladder neck anomaly & $45(21 \%)$ \\
Normal & $19(9 \%)$ \\
Others: & 10 \\
Hypersensitive bladder & 6 \\
Large capacity bladder & 9 \\
Bladder neck obstruction & 3 \\
Urethral stricture & \\
\hline
\end{tabular}

Table 2 Detrusor instability in the group studied

\begin{tabular}{lll}
\hline & $\begin{array}{l}\text { No with } \\
\text { instability }\end{array}$ & $\begin{array}{l}\text { Other abnormalities } \\
\text { in these children }\end{array}$ \\
\hline Boys & 60 & 8 with occult neuropathic bladder \\
& 5 with bladder neck obstruction \\
& 3 with past history of urethral valves \\
& 2 with wide bladder neck anomaly \\
Girls & 73 with wide bladder neck anomaly \\
& 3 with occult neuropathic bladder \\
& 2 with distal urethral stenosis \\
& 1 with vesicovaginal fistula \\
\hline
\end{tabular}

combination being detrusor instability with wide bladder neck anomaly.

As can be seen in Table 2 there were an equal number of boys and girls with detrusor instability, with ages ranging from 3.5 to 16 years and a mean of 8.9 years in girls and 8.7 years in boys.

Forty five children had the wide bladder neck anomaly. Of these, 35 were girls, with ages ranging from 7 to 16 years (mean 10.3 years), seven of whom also had detrusor instability. There were 10 boys with this abnormality, with ages ranging from 8 to 13 years (mean 9.8 years), three of whom had detrusor instability.

\section{Discussion}

Detrusor instability is equally common in boys and girls and is associated with the same type of urge symptoms as in adults. The symptoms tend to improve at puberty and there may be a cause and effect relation with vesicoureteric reflux. ${ }^{6}$ This abnormality was found in $20 \%$ of the children studied who had detrusor instability. Webster et al found reflux in 10 of 28 children with detrusor instability. ${ }^{4}$ Urine infections have been reported to occur in up to $60 \%,{ }^{4-6}$ and we found this association in almost $50 \%$ of children with detrusor instability. None of the chidren, however, had an infection at the time of study.

Urodynamically, the occurrence of unstable con-
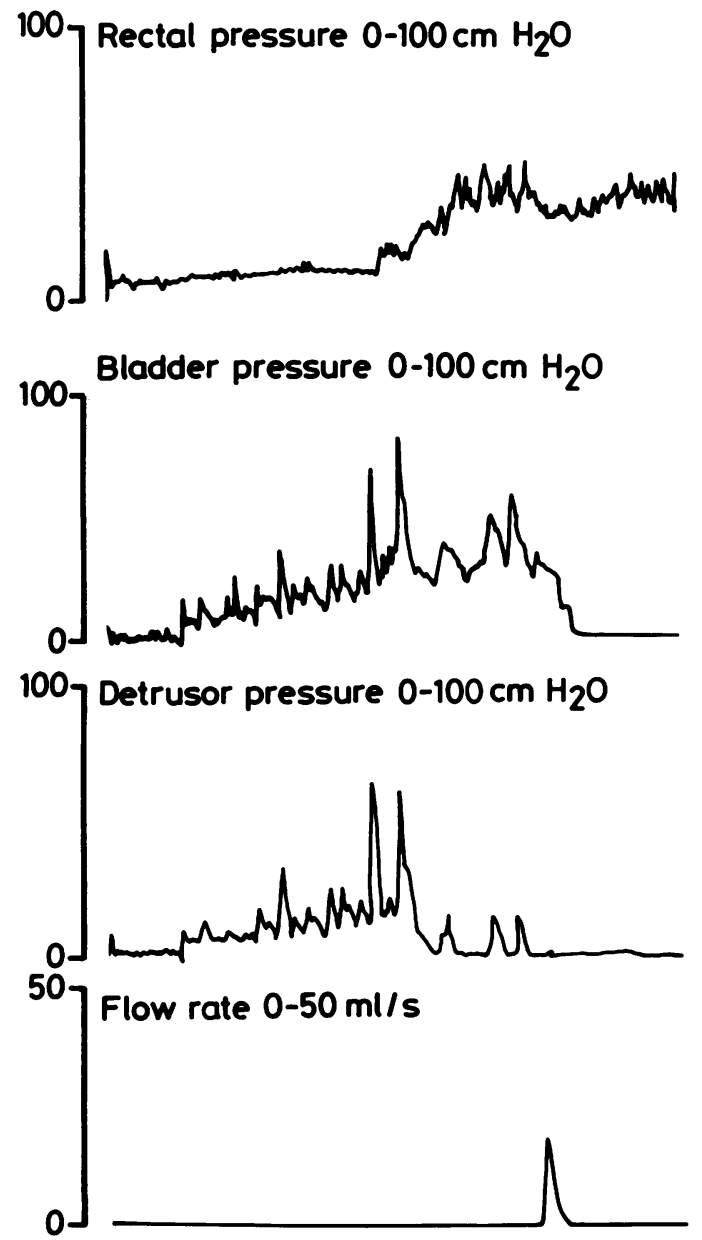

Fig. 1 Cystometrogram, showing unstable detrusor contractions during bladder filling.

tractions is almost always spontaneous, and Figure 1 shows the typical trace obtained in an 8 year old girl who presented with urgency, frequency, and diurnal enuresis. Unstable contractions can be seen shortly after filling begins. These children usually have reduced bladder capacity (capacity $=($ age in years $\times 25)$ $+25 \mathrm{ml}$ ). ${ }^{1}$ Characteristically, the detrusor pressure during filling showed a much more pronounced degree of fluctuation than would be expected in adults with instability. Our finding of this abnormality in just over half of our group agrees with the findings of Webster et al, who found unstable bladders in $47 \%$ of 60 children studied who were neurologically normal but who had micturition dysfunction. ${ }^{4}$ 
Because the pathophysiology of this condition and the transition from incontinent infancy to continent childhood are poorly understood, we have hesitated in making this diagnosis in children under the age of about 5 years. None the less in 'normal' children in this very young age group (with only minor degrees of reflux) it is usual to find a bladder capacity approximating to the formula given above and a fairly 'stable' detrusor pressure until capacity at which time a single 'normal' detrusor contraction produces voiding, albeit 'involuntary' (unpublished observation). The presence of pronounced fluctuations in the detrusor pressure that do not lead to voiding and a reduced bladder capacity are therefore unusual and we think that they represent detrusor instability in this age group.

It is interesting that in younger children instability is almost always spontaneous and the detrusor pressure tends to show low amplitude fluctuations, whereas in older children postural instability is sometimes present and low amplitude fluctuations are less common, as this contrasts with adult practice in which postural or cough induced instability is so much more common, spontaneous instability is less common, and a constantly fluctuating detrusor instability is rare (in the absence of neuropathy). This suggests that the pattern of detrusor behaviour tends to change with time even if the symptomatology does not.

Thirty nine children with detrusor instability had a second study during follow up and 24 had a third. These studies were spaced one to two years apart. During this period the detrusor pressure trace in those that remained unstable tended to become more like that seen in adults with detrusor instability, with discrete phasic waves of pressure interspersed with periods of quiescence.

Incomplete bladder emptying was extremely rare in children with detrusor instability and is therefore unlikely to be related to the increased incidence of urinary infections in this group. It has been suggested, however, that during uninhibited contractions the child voluntarily contracts the external sphincter in an attempt to maintain continence, which increases intravesical pressure, causing mucosal ischaemia, and that this may be a major factor in the development of recurrent infections. ${ }^{4}$

'Milk back' of potentially infected urine may also predispose to infections. Despite the high incidence of infections in this group, impairment of renal function and upper tract problems were rarely seen and had usually been diagnosed when investigating a urinary tract infection, before referral for a videourodynamic study. Children with recurrent urinary infections were treated with prophylactic antibiotics.

Anticholinergic drugs now have an established place in the treatment of detrusor hyperreflexia in association with a neuropathic bladder and have also been used in the treatment of instability in neurologically normal children. They act by decreasing instability and thereby indirectly increasing bladder capacity. We have found these drugs to be useful in the treatment of children with detrusor instability. We have used either propantheline or oxybutynin. Although these results are encouraging, long term follow up is required, and these results will be reported fully at a later date.

In 1973 Stanton and Williams found the wide bladder neck anomaly alone in 21 of 100 children with persistent diurnal incontinence. ${ }^{7}$ They stated that the condition can be recognised by one or more of the following signs: widening of the bladder neck on micturating cystogram; incomplete milk back on micturating cystogram; an open bladder neck on cystoscopy; a low urethral pressure profile; a normal cystometrogram; absence of neurological signs; and absence of distal urethral stenosis. In our experience the condition is associated with a mixture of stress and urge type symptoms, but the presence of stress type symptoms has a strong correlation with the condition in both sexes. It is commoner in girls and tends to improve in puberty in both sexes. It may predispose to genuine stress incontinence in adult life. The abnormality was found more commonly in girls. The diagnosis was made when the bladder neck was seen to be open, throughout the filling phase, at a time when the detrusor was not contracting (Fig. 2). These children may be dry at night.

Our findings of $19 \%$ with this abnormality compares well with $21 \%$ in Stanton and Williams' group. We are currently evaluating the effect of the $\alpha$ adrenergic agonist drug, ephedrine, on this group of children.

Fifteen of the girls had a second videourodynamic study during the follow up and four had a third. None of these girls showed any improvement in

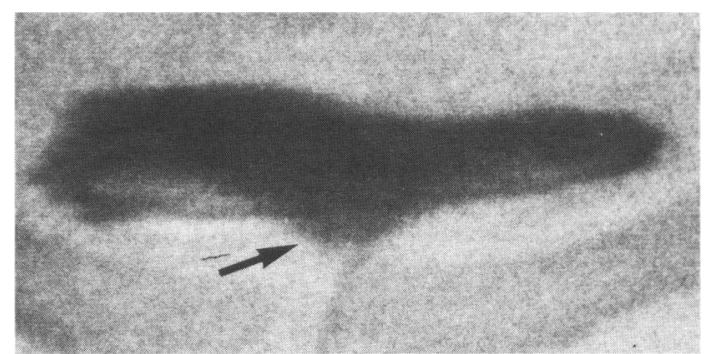

Fig. 2 Photograph taken from videourodynamic study, showing a wide bladder neck (indicated), which was present from the beginning of filling of the bladder. 


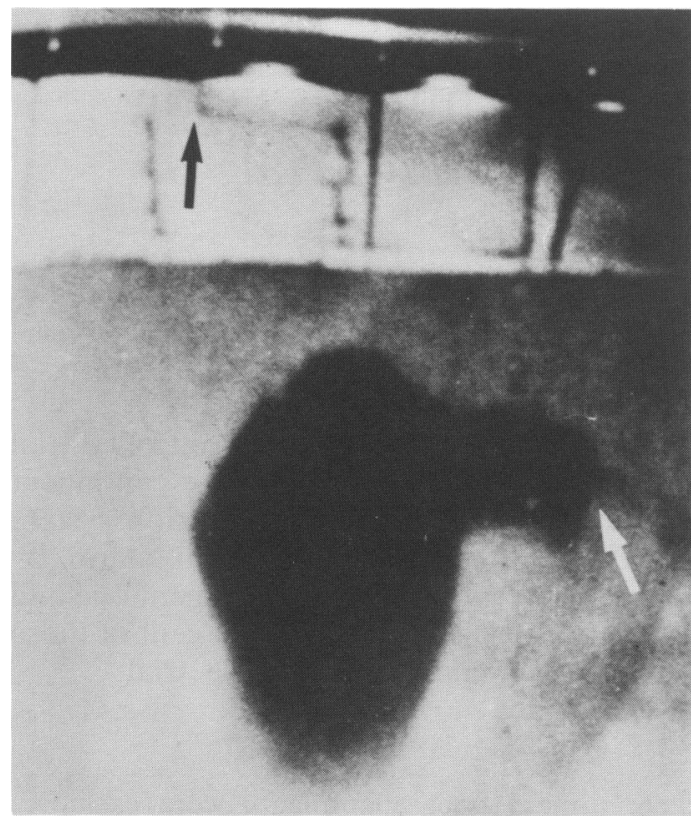

Fig. 3 Photograph taken from a videourodynamic study, showing voluntary contraction of the distal sphincter (white arrow) to prevent urinary leakage during an unstable detrusor contraction (black arrow).

bladder neck competence, even though the symptoms tended to improve at the onset of puberty. Equally, we have yet to see a child who has previously been shown to have a competent bladder neck develop this anomaly.

In our group of 45 children ( 35 girls and 10 boys) seven girls and three boys also had detrusor instability.

The so called 'occult' or 'non-neurogenic' neuropathic bladder was first described by Hinmann and Baumann in $1973^{8}$ and subsequently by other workers ${ }^{9} 10$ and consists of urinary incontinence with some combination of encopresis, heavily trabeculated bladder, urinary infection, vesicoureteric reflux, and a negative neurological examination. All of the investigators noted discoordinate activity between the bladder and external sphincter during detrusor activity. We have certainly seen voluntary detrusor sphincter dyssynergia in children with this problem and this seemed to be a voluntary effort by the child to prevent urine leakage when an unstable contraction occurred (Fig. 3). It may be that the occult neuropathic bladder is an extreme form of an otherwise normal response to unstable detrusor activity.

Soiling in our study group was extremely rare even in the children with an occult neuropathic bladder. One girl with detrusor instability who initially responded well to treatment with drugs subsequently relapsed and started to soil. She was found to have emotional problems related to teasing and underachievement at school and was referred for psychological help.

The third largest group of children in our study (although comprising only $8 \%$ ) were those in whom no abnormality was found. We have found that many of these children simply cannot be bothered to go and void when their bladders are full. For this reason they can be called 'avoiders'. They tend to have social, educational, or emotional problems and most can be diagnosed clinically. Psychological problems may coexist with abnormal videourodynamic findings, and the present study was not designed to look at this relation nor is it concluded that treatment with drugs alone is all that is required. A primarily psychological style of management may well be incomplete, however, in terms of both information and possible treatment.

An important finding in our study was that only $8 \%$ of the children had normal studies, and this emphasises the importance of investigating these children. This is best performed by carrying out a combined videourodynamic study. These children cannot be accurately diagnosed on the basis of symptomatology alone. Some have more than one abnormality and this may be missed if a combined study is not undertaken. Finally, we think that many of these children can be helped if accurately assessed, although long term follow up is necessary.

\section{References}

${ }^{1}$ Mundy AR, Borzyskowski M, Saxton HM. Videourodynamic evaluation of neuropathic vesicourethral dysfunction in children. Br J Urol 1982;54:645-9.

${ }^{2}$ Whiteside CG. Videocystographic studies with simultaneous pressure and flow recordings. Br Med Bull 1972;28:214-9.

3 Whiteside CG, Arnold EP. Persistent primary enuresis - a urodynamic assessment. Br Med J 1975;i:364-7.

${ }^{4}$ Webster GD, Koefoot RB Jr, Sihelnik S. Urodynamic abnormalities in neurologically normal children with micturition dysfunction. J Urol 1984;132:74-7.

${ }^{5}$ Firlit CF, Smey P, King LR. Micturition urodynamic flow studies in children. J Urol 1978;119:250-3.

${ }^{6}$ Koff SA, Lapides J, Piazza DH. Association of urinary tract infection and reflux with uninhibited bladder contractions and voluntary sphincteric obstruction. J Urol 1979;122:373-6.

${ }^{7}$ Stanton L, Williams DI. The wide bladder neck in children. $\mathrm{Br}$ J Urol 1973;45:60-4.

${ }^{*}$ Hinmann F, Baumann FW. Vesical and uretheral damage from voiding dysfunction in boys without neurological or obstructive disease. J Urol 1973;109:727-32.

' Allen TD, Bright TC. Urodynamic patterns in children with dysfunctional voiding problems. J Urol 1978;119:247-9.

10 Firlit CF, Smey P, King LR. Micturition urodynamic flow studies with children. J Urol 1978;119:250-3.

Correspondence to Dr M Borzyskowski, Newcomen Centre, Guy's Hospital, London, SE1 9RT.

Received 24 July 1986 